\title{
Alterations in Three-Dimensional Organization of the Cancer Genome and Epigenome
}

\author{
Joanna Achinger-Kawecka, ${ }^{1,2}$ Phillippa C. Taberlay, ${ }^{1,2,3}$ and Susan J. Clark ${ }^{1,2}$ \\ ${ }^{1}$ Epigenetics Research Laboratory, Genomics and Epigenetics Division, Garvan Institute of Medical \\ Research, Darlinghurst, New South Wales 2010, Australia \\ ${ }^{2}$ St. Vincent's Clinical School, Faculty of Medicine, University of New South Wales, Darlinghurst, \\ New South Wales 2010, Australia \\ ${ }^{3}$ School of Medicine, Faculty of Health, University of Tasmania, Hobart, Tasmania 7000, Australia \\ Correspondence: s.clark@garvan.org.au
}

\begin{abstract}
The structural and functional basis of the genome is provided by the three-dimensional (3D) chromatin state. To enable accurate gene regulation, enhancer elements and promoter regions are brought into close spatial proximity to ensure proper, cell type-specific gene expression. In cancer, genetic and epigenetic processes can deregulate the transcriptional program. To investigate whether the $3 \mathrm{D}$ chromatin state is also disrupted in cancer we performed Hi-C chromosome conformation sequencing in normal and prostate cancer cells and compared the chromatin interaction maps with changes to the genome and epigenome. Notably, we find that additional topologically associated domain (TAD) boundaries are formed in cancer cells resulting in smaller TADs and altered gene expression profiles. The new TAD boundaries are commonly associated with copynumber changes observed in the cancer genome. We also identified new cancer-specific chromatin loops within TADs that are enriched for enhancers and promoters. Finally, we find that many of the long-range epigenetically silenced (LRES) and longrange epigenetically active (LREA) regions in cancer are characterized by differential chromatin interactions. Together our data provide a new insight into charting alterations in higher-order structure and the relationship with genetic, epigenetic, and transcriptional changes across the cancer genome.
\end{abstract}

The genome is not simply linear, but is exquisitely folded into a three-dimensional (3D) chromatin structure to facilitate appropriate cell type-specific gene expression patterns. The epigenome partitions the genome into active and inactive domains. Active regions are marked by open chromatin and active histone marks, whereas inactive regions are marked by repressive histone modifications and closed chromatin (Fig. 1). For a gene to be active there needs to be a physical interaction or chromatin interaction that enables genomic regions that are distant in linear space on chromosomes to come in close proximity in 3D space and relay signals-for example, via enhancer-promoter looping interactions (Fig. 1). Chromatin loops are maintained by architectural proteins - for example, insulator protein CCCTC-binding factor (CTCF) and SMC-family complex, cohesion (RAD21) (Dixon et al. 2012; Nora et al. 2012; Rao et al. 2014). Rapid advances of chromosome conformation capture (3C)-based (Dekker et al. 2002) technologies such as 4C (Simonis et al. 2006; Zhao et al. 2006), 5C (Dostie et al. 2006), Hi-C (Lieberman-Aiden et al. 2009), and ChIA-PET (Fullwood et al. 2009) have provided increasing insights into the $3 \mathrm{D}$ architecture of the genomes. It is now clear that the genome is organized into topologically associated domains (TADs) or contact domains that are hundreds of kilobases in size and encompass multiple genes and regulatory elements (Dixon et al. 2012; Nora et al. 2012). At a higher level, TADs are organized into functionally distinct megabase compartments, which comprise large blocks of chromatin that are either A-type (open, gene-rich) or B-type (closed, genepoor) (Lieberman-Aiden et al. 2009; Zhang et al. 2012). TADs are characterized by preferential interactions within them and lack of interactions between different TADs, suggesting that TAD boundaries act as insulators by preventing communication between elements on either side of the boundary. TADs are conserved and reported to be largely invariant between different cell types (Dixon et al. 2012; Nora et al. 2012), whereas the chromatin interactions or loops within TADs are more tissue-specific (Dixon et al. 2012, 2015; Phillips-Cremins et al. 2013; Smith et al. 2016). Because the genome and the epigenome are both highly deregulated in many cancer types, we were interested to determine whether the $3 \mathrm{D}$ chromatin organization in cancer was also disrupted and if this was associated with the aberrant gene expression, such as activation of oncogenes or inactivation of tumor-suppressor genes.

\section{TAD ORGANIZATION IS DISRUPTED IN PROSTATE CANCER CELLS}

To elucidate the role of $3 \mathrm{D}$ genome architecture in prostate cancer biology, we performed Hi-C and RNA-seq experiments in normal prostate epithelial cells (PrECs)

(C) 2016 Achinger-Kawecka et al. This article is distributed under the terms of the Creative Commons Attribution License, which permits unrestricted reuse and redistribution provided that the original author and source are credited. 


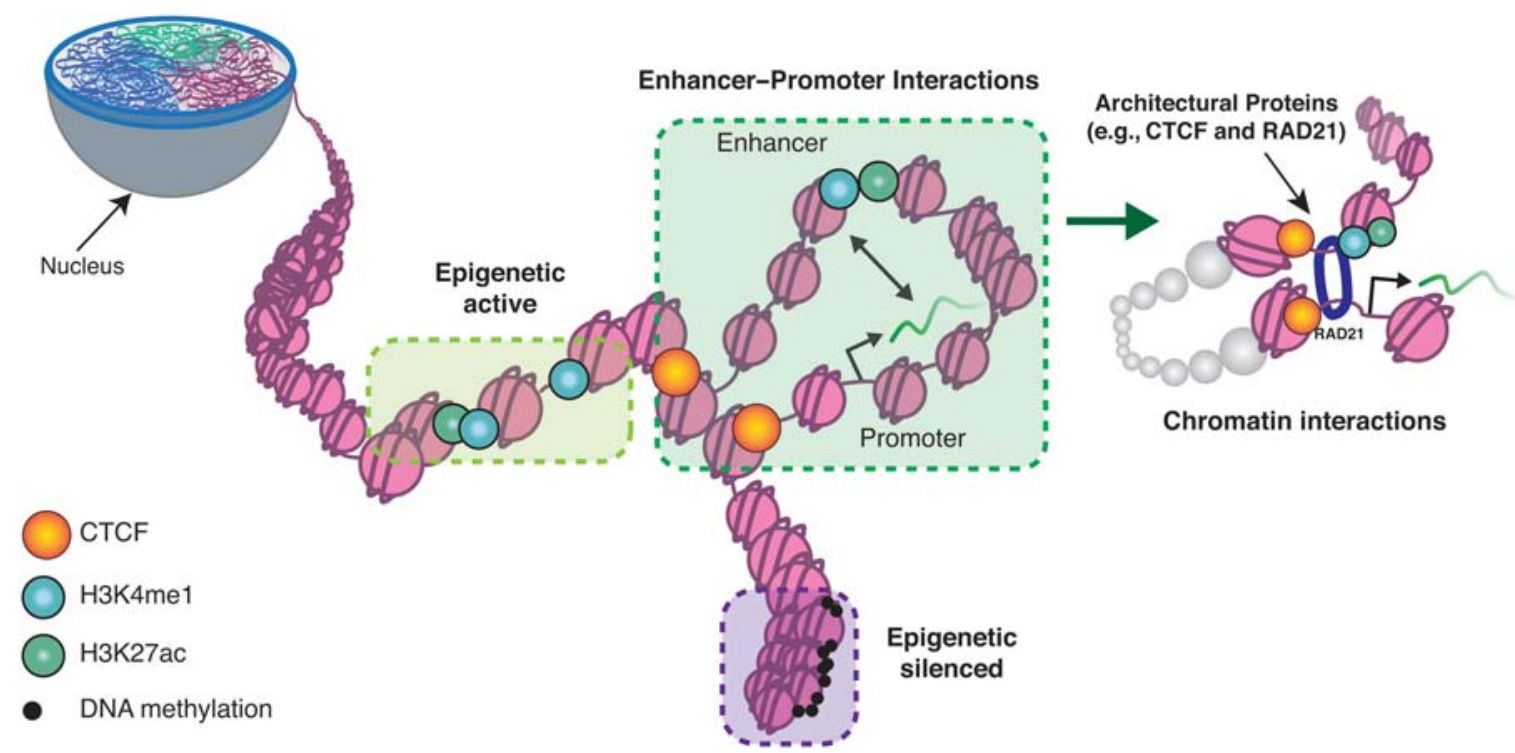

Figure 1. The epigenome partitions the genome into inactive or active domains. Inactive regions are marked by closed chromatin and repressive modifications (e.g., DNA methylation) and active regions are marked by open chromatin and active histone marks (e.g., $\mathrm{H} 3 \mathrm{~K} 4 \mathrm{me} 1$ and $\mathrm{H} 3 \mathrm{~K} 27 \mathrm{ac}$ ). For a gene to be active there needs to be a physical chromatin interaction that enable genomic regions that are distant in a linear space on chromosomes to come in close proximity in three-dimensional space and relay signals (e.g., via enhancer-promoter interactions). These have been shown to be maintained by architectural proteins (e.g., CTCF and RAD21).

and two prostate cancer cell lines (LNCaP, androgensensitive, and PC3, androgen-resistant) (Taberlay et al. 2016). To further understand the relationship between cancer genome organization and genetic and epigenetic remodeling, we used ChIP-seq to probe binding patterns of transcription factors CTCF and RAD21 and regulatory elements, including enhancers (H3K4me1 and H3K27ac), promoters (H3K4me3), and Polycomb (H3K27me3).

Considering the importance of the topological organization of the genome, we next explored the segmentation of the normal and cancer genomes into TADs (Dixon et al. 2012; Nora et al. 2012). Changes within TADs (sub-TADs) have been reported to occur during cell differentiation (Dixon et al. 2015) and reprogramming (Beagan et al. 2016; Krijger et al. 2016) in embryonic stem cells, which suggests that within the hierarchical genome structure there is a discrete level of $3 \mathrm{D}$ organization that displays more plasticity to facilitate cell type-specific expression. We hypothesized that TAD organization would be disrupted in cancer cells and potentially resemble more closely the organization observed in undifferentiated cells. Comparison of the TAD organization in normal prostate cells and prostate cancer cells revealed that cancer cells retain the ability to segment their genomes into domains; however, we observed large differences in both their number (Fig. 2A) and in the position of the TAD boundaries across the genome (Fig. 2B). The size of TADs was significantly smaller in the two prostate cancer cell lines compared with normal cells. In PC3 cells, TADs were on average half the size of TADs in normal PrECs and the number of TADs increased by approximately twofold compared with PrECs (Fig. 2A). Interestingly, the large increase in the number of TADs and the decrease in their average size were even more prominent in $\mathrm{LNCaP}$ cells. In $\mathrm{LNCaP}$ cells, TADs were on average 0.3 times the size of those in PrECs and their number increased by 3.5 -fold (Fig. 2A). The variability between the two prostate cancer cell lines could be potentially explained by the differences in the biology of these cells.

By visualizing topological domains as $2 \mathrm{D}$ interaction matrices, we observed that large domains in normal cells were often subdivided into smaller domains ("subdomains") in cancer cells (Fig. 2B). We further validated this observation, by comparing the number of TADs identified in cancer cells that are located entirely within the large TADs observed in PrECs to a random overlap. Indeed, the number of cancer-associated domains (identified in PC3 and LNCaP cells) found to be subdomains of normal cell (PrEC) TADs was significantly higher than expected by chance $(P<0.01)$. Similar observations were recently reported by Barutcu et al. $(2015,2017)$. Here the authors studied genome-wide chromatin interaction patterns in epithelial and breast cancer cells and discovered that TADs were frequently "broken" into multiple sub-TADs in cancer cells. Taken together, the findings show that although the segmentation of the genome into well-defined TADs is still maintained in the cancer genome, the characteristics of TADs in cancer cells are significantly different to TADs in normal cells.

To further delineate the differences we observed in the TAD organization between normal and cancer genomes, we intersected the TAD boundaries identified in each of the cell types and categorized the boundaries as either "constitutive" (present in all three cell types), "cancerspecific" (present in both cancer cell lines, but not in normal cells), or "facultative" (maintained in only one of the cell lines studied). Topological domain boundaries 
A

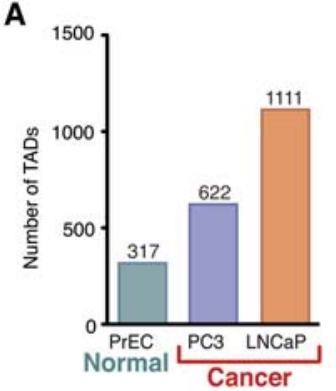

C

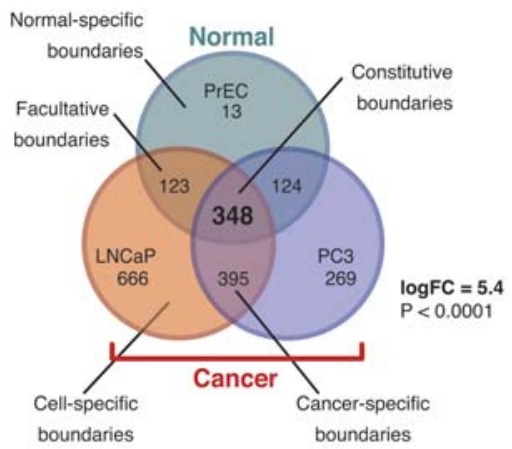

D

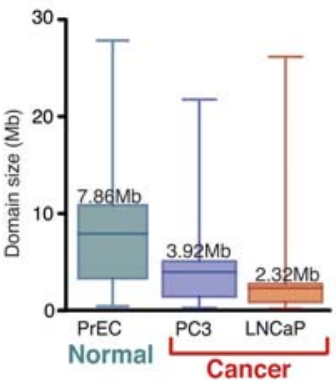

B

\section{(IIIIIIIIIIII) IIIIIIIIII \\ $5 \mathrm{Mb} \longmapsto$ hig 19 \\ $55,000,000$ | $58,000,000$ | $61,000,000$ | $64,000,00$ ㅇ $67,000,000$ | $70,000,000$ \\ PrEC \\ Normal}
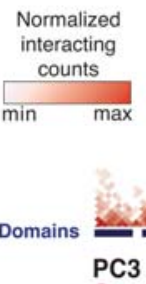

PC3

Cancer

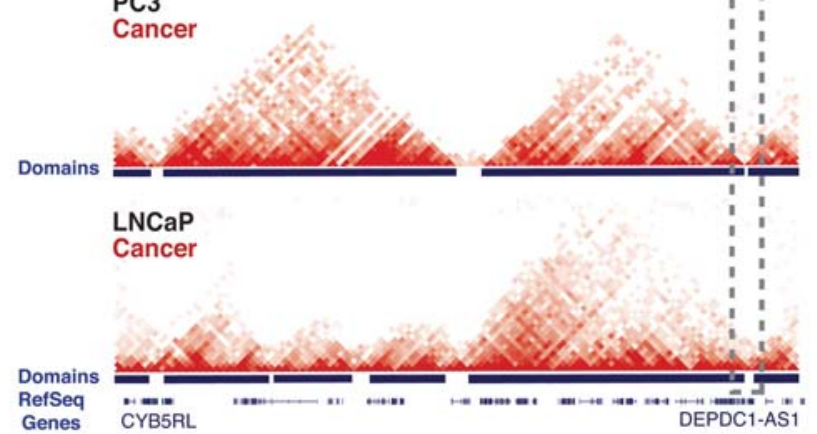

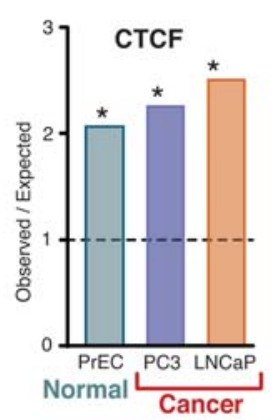

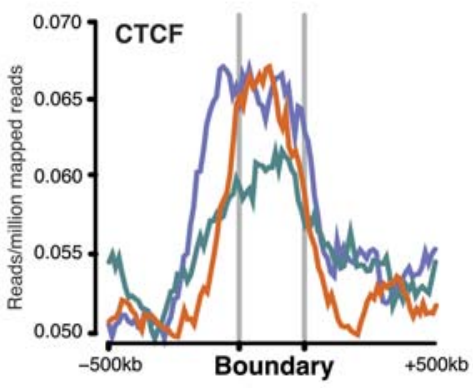

PrEC (Normal)

PC3 (Cancer) $\square$ LNCaP (Cancer)

Figure 2. Topologically associated domains (TADs) in the cancer cell genome. $(A)$ Number and average size (in Mb) of domains identified in normal prostate cells (PrEC) and prostate cancer cells (PC3 and LNCaP). (B) An example from chromosome 1 of a twodimensional chromatin interaction matrix from PrEC, PC3, and LNCaP cells visualized in the WashU Epigenome Browser. Large TADs found in normal cells are frequently subdivided into two or more domains in cancer cells. $(C)$ The majority of TAD boundaries present in normal cells (PrEC) are also present in cancer cells (constitutive boundaries), whereas $\sim 20 \%$ of boundaries were maintained in only one of the two cancer cells (cell type-specific boundaries). Cancer cells (LNCaP and PC3) share a large proportion of their TAD boundaries (cancer-specific boundaries; $\log \mathrm{FC}=5.4, P<0.0001)$. $(D)$ Observed/expected fold enrichment of CTCF binding at the domain boundaries in PrEC, PC3, and LNCaP cells. Genome-wide average distribution of CTCF binding around the TAD boundaries in PrEC, PC3, and LNCaP cells. (Adapted, with permission, from Taberlay et al. 2016, C Cold Spring Harbor Laboratory Press.)

represent the region of strict division between two TADs and are characterized by high stability among normal cell types (Dixon et al. 2012, 2015). Epigenetic marks, gene expression, and replication timing were previously shown to be regulated by TAD architecture (Le Dily et al. 2014; Pope et al. 2014). Recent Hi-C studies revealed that architectural proteins play a critical role in determining the $3 \mathrm{D}$ organization of the genome. The insulator proteins, CTCF and cohesin, are the most well-known architectural proteins that are known to be involved in TAD formation and maintenance (Misteli 2007; Phillips and
Corces 2009). However, the exact mechanisms by which architectural proteins function to form and maintain TAD boundaries are still not well-understood. In multiple cell types, TAD boundaries have been shown to work as insulators by restricting interaction with genes in the neighboring TADs (Nora et al. 2012; Andrey et al. 2013; Symmons et al. 2014; Giorgio et al. 2015; Lupianez et al. 2015; Tsujimura et al. 2015; Franke et al. 2016).

Thus, we were prompted to investigate what factors might be contributing to the loss of TAD boundary integrity in cancer. Comparing the locations of boundaries 
between the two prostate cancer cell lines, we found $>90 \%$ of boundaries present in normal cells were also present in at least one cancer cell type (Fig. 2C). Of these, $\sim 80 \%$ were "constitutive" (present in all three cell types), whereas $\sim 20 \%$ were "facultative," as they were maintained in only one of the two cancer cell lines studied. Importantly, out of all of the nonconstitutive boundaries, $30 \%$ were cancer-specific TAD boundaries. This suggests that the acquisition of new sub-TAD boundaries at similar genomic locations is a cancer-specific process of $3 \mathrm{D}$ reorganization. In support of this concept, we observed high fold change (observed over expected) overlap between the two sets of new TAD boundaries in each cancer cell type (Fig. 2C). An intriguing explanation for the formation of new TAD boundaries in cancer is that these may be related to widespread epigenome remodeling and genetic alterations that are frequently observed in cancer. To test that possibility, we analyzed ChIP-seq data of architectural proteins and histone modifications. We found that TAD boundaries in all three cell types were highly enriched for CTCF (Fig. 2D), with $\sim 7 \%$ of all CTCF binding sites being located at TAD boundaries. However, we did not observe major differences in CTCF enrichment between normal and cancer cells. This result may be expected because CTCF is known to be a conserved insulator element (Li et al. 2013).

Recently, there has been a growing interest into elucidating the role of genetic changes on the organization of the $3 \mathrm{D}$ chromatin in cancer. The vast majority of genetic alterations found in the cancer genome occur outside of protein coding regions, and therefore the functional consequences can be difficult to interpret. We hypothesized that alterations frequently found in the cancer genomes may result in the disruption of TADs and therefore provide an explanation for the large increase in sub-TADs observed in the cancer cells. It is well established that the genomes of prostate cancer cells can have recurrent copynumber alterations (Williams et al. 2014; Boutros et al. 2015). We therefore next asked whether copy-number variants (CNVs) could provide a compelling explanation for the formation of new TAD boundaries. We surmised that copy-number changes located within a TAD could result in change in local chromatin interactions and thus lead to aberrant contacts between two distant regions and formation of a new TAD boundary. To test this hypothesis, we identified 60 chromosomal variants, up to $10 \mathrm{Mb}$ in size, that were present in both LNCaP and PC3 cells, identified using single-nucleotide polymorphism (SNP) arrays. We intersected the locations of cancer-specific TAD boundaries with the identified cancer CNVs. Interestingly, we observed that $\sim 70 \%$ of the CNVs that were shared between LNCaP and PC3 cells were also located at or in close proximity to the newly formed cancer-specific domain boundaries. For example, a 400- to 600-kb cancer-specific deletion on Chromosome 17p13.1 is associated with the establishment of a new domain boundary in both prostate cancer cell lines and a resulting change in local interactions across this region (Fig. 3). Interestingly, this locus harbors the TP53 gene and is commonly deleted $(14.8 \%)$ in all prostate cancers (Kluth et al. 2014). Consistent with this finding, previous reports suggested that genomic deletions at TAD borders in normal cells result in a change in local chromatin organization and in a formation of new domain boundaries (Nora et al. 2012; Ibn-Salem et al. 2014). These findings suggest an important role of genetic alterations in defining the cancer-specific disruption to the 3D genome organization. As it is known that TAD boundaries are crucial for controlling the action of enhancers on genes in adjacent TADs (Nora et al. 2012; Dowen et al. 2014; Symmons et al. 2014), our findings may help explain the altered gene expression profiles in cancer cells.

\section{CANCER CELLS ARE CHARACTERIZED BY FREQUENT LOCAL DIFFERENTIAL CHROMATIN INTERACTIONS}

Alterations in local chromatin interactions have been a focus of many studies into long-range gene regulation and oncogene activation in cancer. To further understand the role of atypical chromatin interactions in prostate cancer, we used a diffHiC package (Lun and Smyth 2015) to identify and characterize differential interactions between normal prostate and prostate cancer cells. diffHiC permits analysis of interactions with significant differences in intensity between two biological conditions. Then we used the diffHiC data to define anchor points of chromatin interactions (Fig. 4A). By comparing normal prostate (PrEC) interaction data independently to each of the prostate cancer data sets (PC3 and LNCaP), we obtained more than 2000 differential high-confidence interactions for each of the comparisons (Fig. 4B). By intersecting the two differential data sets, we found that majority of all differential interactions identified in a comparison of PrEC with PC3 were also found in a comparison of PrEC with LNCaP, suggesting that the majority are common, cancer-specific interactions (Fig. 4B). Interestingly, differential interactions were enriched in cancer cells, suggesting that cancer cells gain interactions more easily than lose interactions (Fig. 4C). This observation is in agreement with previous reports showing that cancer cells have atypical enhancer-promoter interactions that lead to disruption of gene regulation (for review, see AchingerKawecka and Clark 2017). Dixon et al. (2015) recently showed that majority of changes in local chromatin interactions during cell differentiation occur within the borders of the TADs, with only a very small number of interactions crossing the TAD boundaries. This suggests that atypical enhancer-promoter interactions in cancer cells may be restricted by the presence of conserved TAD boundaries in these cells. Notably, we observed that the most significant differential interactions are predominantly located within topological domains, further supporting that hypothesis.

To delineate the role of differential interactions in prostate cancer biology, it is crucial to understand their potential context in relation to epigenome remodeling. To determine whether regulatory elements may be associated with differential interactions, we used ChromHMM 

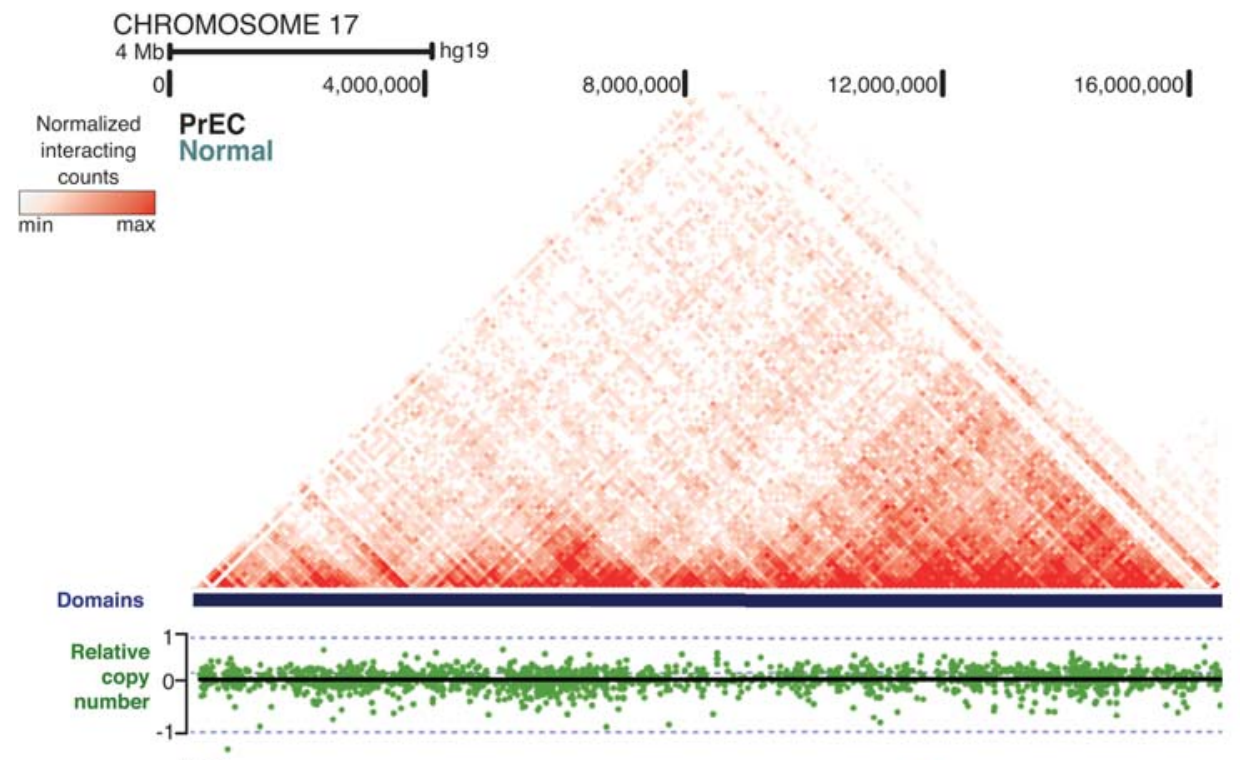

PC3
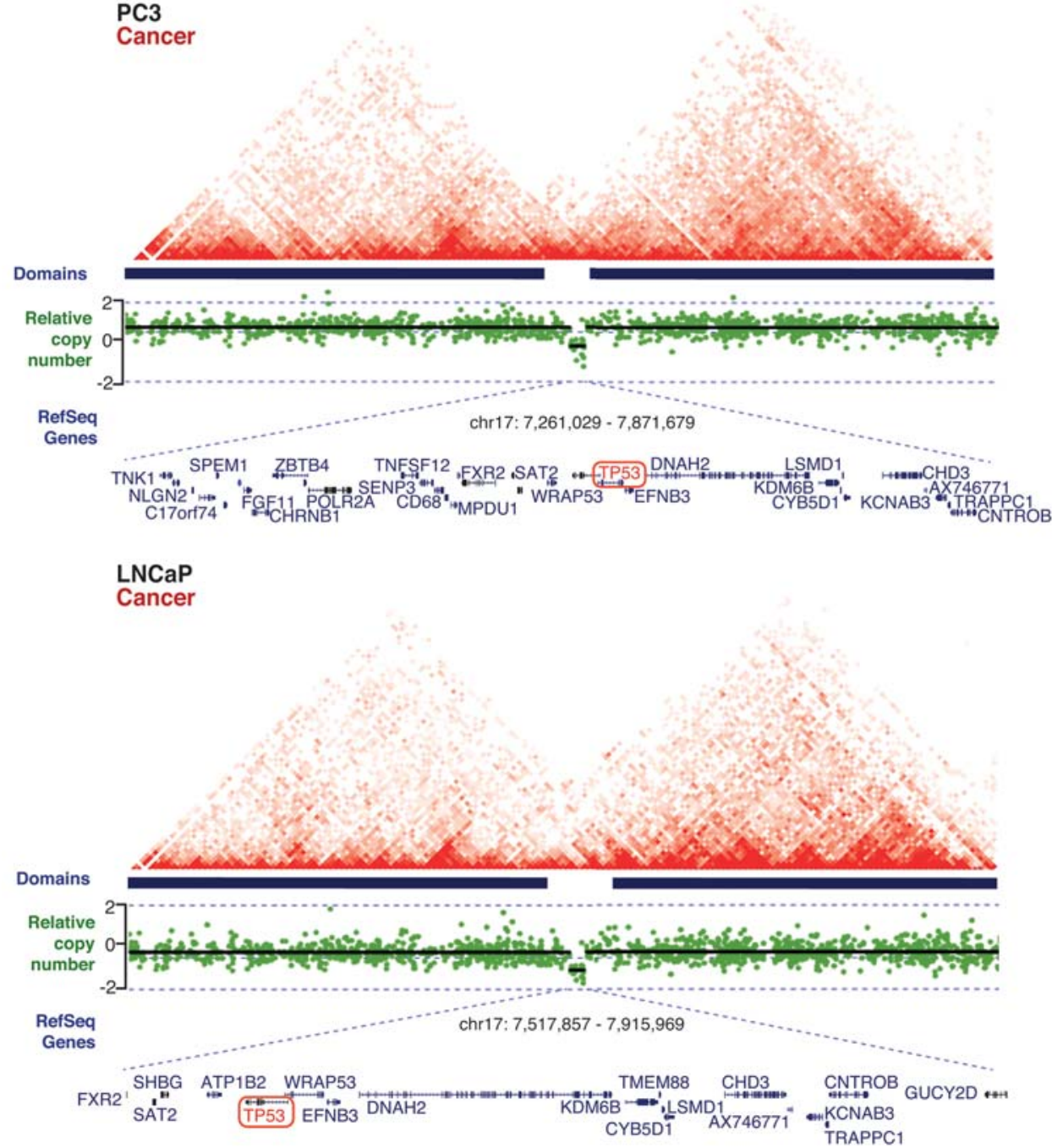

Figure 3. Copy-number variants (CNVs) are located at cancer-specific topologically associated domain (TAD) boundaries. A representative example from Chromosome 17 showing a deletion encompassing the TP53 locus (shown in green) that is present in both cancer cell lines is associated with a formation of a new, cancer-specific domain boundary and a change in local chromatin interactions in both cancer cell lines. (Reprinted, with permission, from Taberlay et al. 2016, C Cold Spring Harbor Laboratory Press.) 

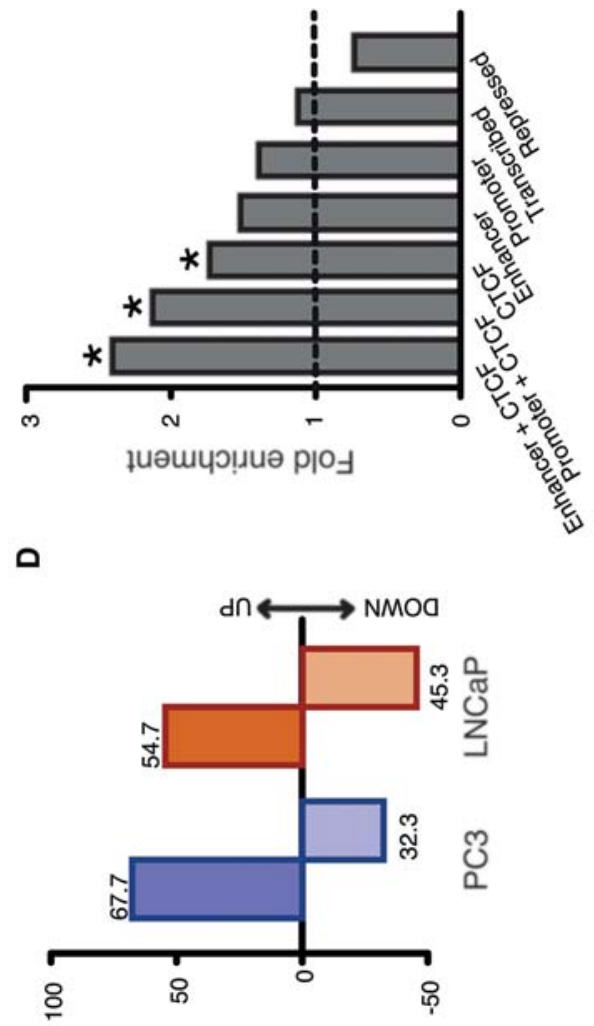

(\%) ৩ $\exists^{\perp d}$

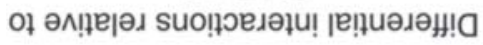

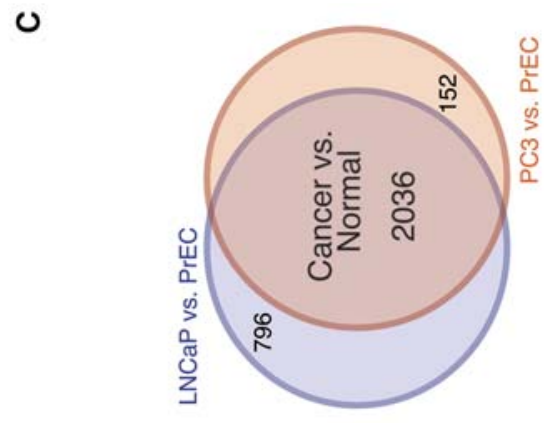

m

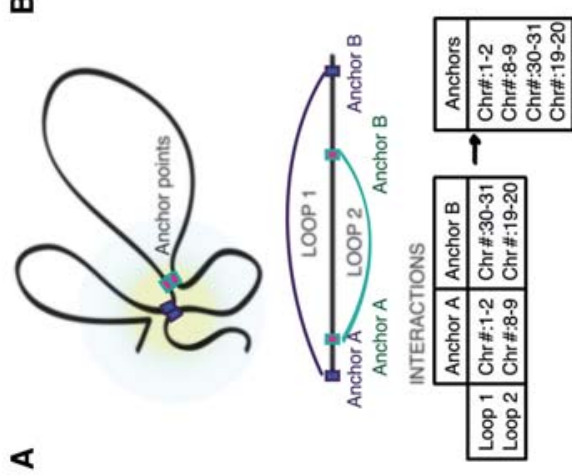

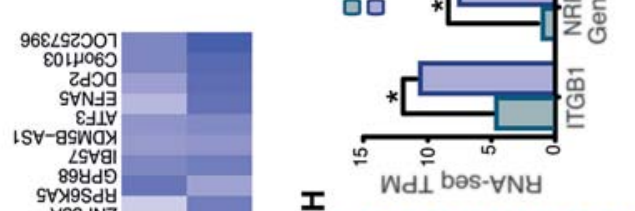

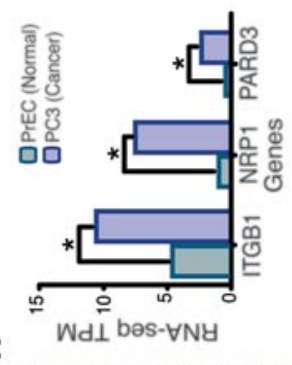

$\forall E \& \exists N$

$10 \mathrm{da}$

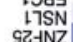

SZINZ

cocate

घอWHY

$\angle \mathrm{OAW}$

zsग्)

twdyI

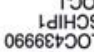

$96 t \in N Z$
$7 \mathrm{dN} 30$

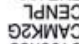

squoolo

캐슬

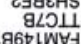

286tiWY

69 나닌

ztdSno

$2 S \forall \forall O$
$t \rightarrow \forall O C$

ษELEW

เาตเอม

9ygn
Xoघg

60 IVNO

IWEd

ssาd

8SWOY

$2007 d$

SU甘7

$105 \mathrm{~A}$
$\forall 9 z S d \wedge$

$17 \mathrm{dOS}$

INGS

x y

เมติ

กำ

6W

IVXNY

I $W W N O Y$

Logjd

9เSdYW

\&dOISdy

\&WNN

เN70ดก

เมูบร

IdXYd

ZIN7Y

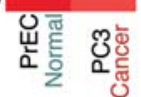

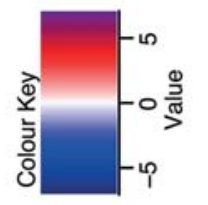

ш
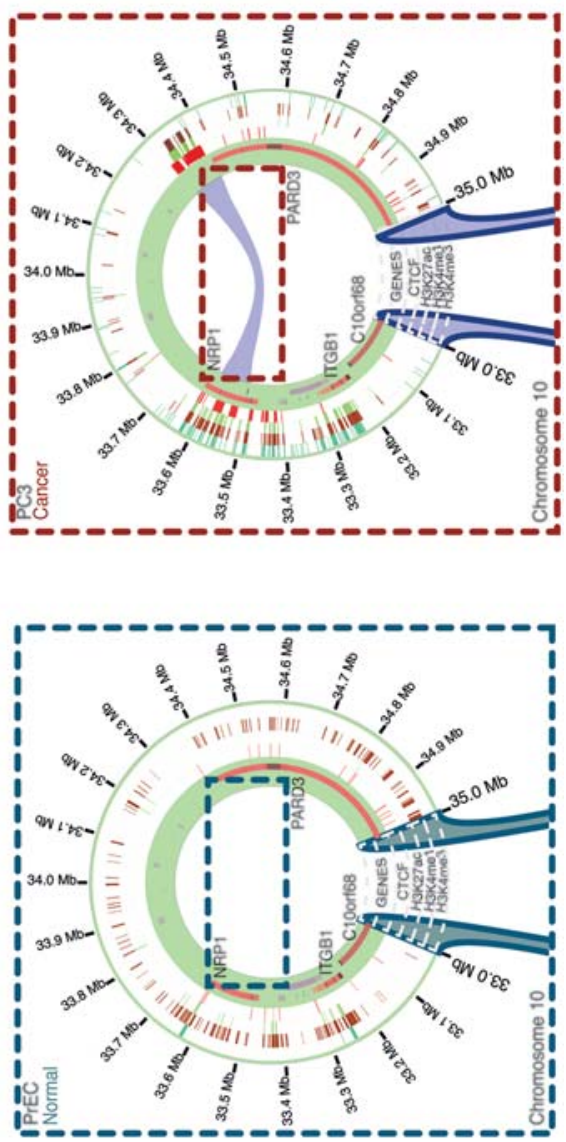

v

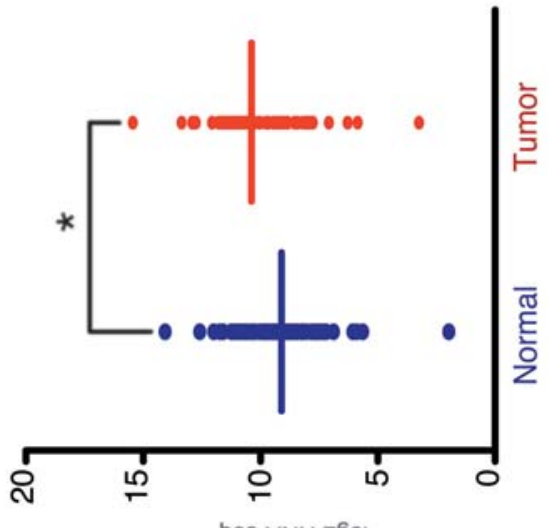

น

bəs- $\forall N Y$ Z60। 
(Ernst and Kellis 2012) and annotated the epigenomes of each normal and cancer cell type into distinct chromatin states (obtained from Taberlay et al. 2014). When we intersected the identified chromatin states with the anchor points of cancer-specific interactions, we observed high enrichment for enhancers that were marked by CTCF, promoters marked by CTCF as well as distal CTCF sites, suggesting that functional elements occupied by CTCF may facilitate new interactions in cancer cells (Fig. 4D). The high overrepresentation of enhancers and promoters at differentially interacting sites prompt us to investigate whether changes in local chromatin interactions could affect expression of nearby genes. First, we compared the expression of genes located at anchor points of differential interactions between normal and cancer cells using RNA-seq and we discovered that novel cancer-specific chromatin interactions were associated with altered gene expression (Fig. 4E). Altogether, $\sim 80 \%$ of all differential interactions in cancer cells correlated with differential expression of genes located at anchor points (Fig. 4E), which is consistent with their enrichment for enhancers and promoters. Second, we hypothesized that genes located at cancer-specific interactions may be involved in the prostate cancer pathology, and therefore their expression would be significantly disrupted in tumor samples from prostate cancer patients. To validate, we tested whether expression of genes located within differential interactions was also altered in the TCGA prostate cohort (Cancer Genome Atlas Research Network 2015). In support of this concept, we determined that expression of more than half of all genes located at differential interactions was significantly increased in tumor samples compared with normal prostate samples (Fig. 4F). It is interesting to speculate that functional elements, especially ones occupied by CTCF, may facilitate new chromatin interactions in cancer cells. Enhancers are known to engage in interactions that exist in a cell type-specific manner and our results support the hypothesis that enhancer dynamics play a role in regulating local interaction differences between normal and cancer cells. Furthermore, altered chromatin interactions may lead to deregulation of gene expression and contribute to increased expression of oncogenes in cancer cells and repression of tumor-suppressor genes.

Our findings highlight that differential chromatin interactions are enriched at enhancers and promoters; however, these potential new 3D loops remain difficult to visualize in a linear genome browser. With this in mind we developed Rondo, a web-based interface for visualizing Hi-C data (Taberlay et al. 2016). Rondo displays gene tracks and epigenome data sets, such as ChIP-seq and RNA-seq, allowing biological interpretation of Hi-C data in context of other "omic" data sets. Using Rondo, we visualized differential interactions alongside histone modifications, and then intersected these with gene expression data to determine the functional output of atypical interactions in the prostate cancer cells. A representative example of a cancer-specific interaction, which involves a change in enhancer and promoter marks, as well as in gene expression, is presented in Figure 4G. In cancer cells, a novel loop between the enhancer and promoter of NRP1 results in regional activation of genes as shown by RNA-seq (Fig. 4H). In normal prostate cells $N R P 1$ is not active, and the putative enhancer is marked by residual $\mathrm{H} 3 \mathrm{~K} 4 \mathrm{me} 1$ but is devoid of $\mathrm{H} 3 \mathrm{~K} 27 \mathrm{ac}$, $\mathrm{H} 3 \mathrm{~K} 4 \mathrm{me} 3$, and CTCF binding in normal cells. In cancer cells, a marked increase in $\mathrm{H} 3 \mathrm{~K} 4 \mathrm{me} 1, \mathrm{H} 3 \mathrm{~K} 4 \mathrm{me} 3$, and $\mathrm{H} 3 \mathrm{~K} 27 \mathrm{ac}$ signal is observed at both the putative enhancer and promoter regions of the gene, together with increase in CTCF binding. Remarkably, this epigenetic remodeling was not restricted to the NRP1 gene boundaries and also encompassed neighboring enhancers and cancerassociated genes, such as ITGBI and PARD3. Expression of ITGB1, NRP1, and PARD3 genes were all significantly increased in the cancer cells (Fig. 4H), suggesting that functional elements and long-range epigenetically regulated domains may be altered concomitant with the interactome. Interestingly, NRPI has been shown to participate in several different types of signaling pathways that control cell migration and overexpression of NRP1 has been reported to predict distant relapse after radical prostatectomy in clinically localized prostate cancer (Talagas et al. 2013).

\section{RELATIONSHIP BETWEEN LONG-RANGE EPIGENETIC REMODELING AND 3D CHROMATIN ORGANIZATION}

The concordant inactivation of adjacent genes due to long-range epigenetic silencing (LRES) has been reported in various cancer types, including colorectal, bladder, non-small cell lung cancer, breast, prostate, and Wilms' tumor (Frigola et al. 2006; Stransky et al. 2006; Hitchins Figure 4. (Figure on previous page.) Differential interactions observed in cancer cells are enriched for enhancers and promoters and
are coincident with altered cancer epigenetic patterns. $(A)$ Anchor points of chromatin interactions were defined as the genomic
locations where an interaction is present. $(B)$ Overlap between anchor points of differential interactions between PrEC and PC3 and
between PrEC and LNCaP cell lines. $(C)$ Differential interactions are enriched in cancer cells. $(D)$ Enrichment of differential
interactions for enhancers that were marked by CTCF (enhancer + CTCF), promoters marked by CTCF (promoter + CTCF), as
well as distal CTCF sites (CTCF) using ChromHMM. $(E)$ Differential interactions are associated with significantly altered gene
expression in cancer cells. $(F)$ Genes located at differential interactions are associated with altered expression in primary prostate
cohort (TCGA PRAD) ( $\log _{2}$ RNA-seq, $P$ value $\left.<0.001\right)$. ( $G$ ) A representative example from Chromosome 10 showing epigenetic
remodeling (activation) of genes located at the differentially interacting region in cancer cells. The promoter and a putative enhancer of
the NRP1 gene are both marked by residual H3K4me 1 in normal cells. In cancer cells there is a marked increase in H3K4me1,
H3K4me3, and H3K27ac in cancer cells, indicative of increased gene activity that is association with a formation of a new cancer-
specific interaction. $(H)$ Genes located at differential interaction are significantly overexpressed in cancer cells $\left({ }^{*} q\right.$ value $\left.<0.0001\right)$.
(Adapted, with permission, from Taberlay et al. 2016, C) Cold Spring Harbor Laboratory Press.) 

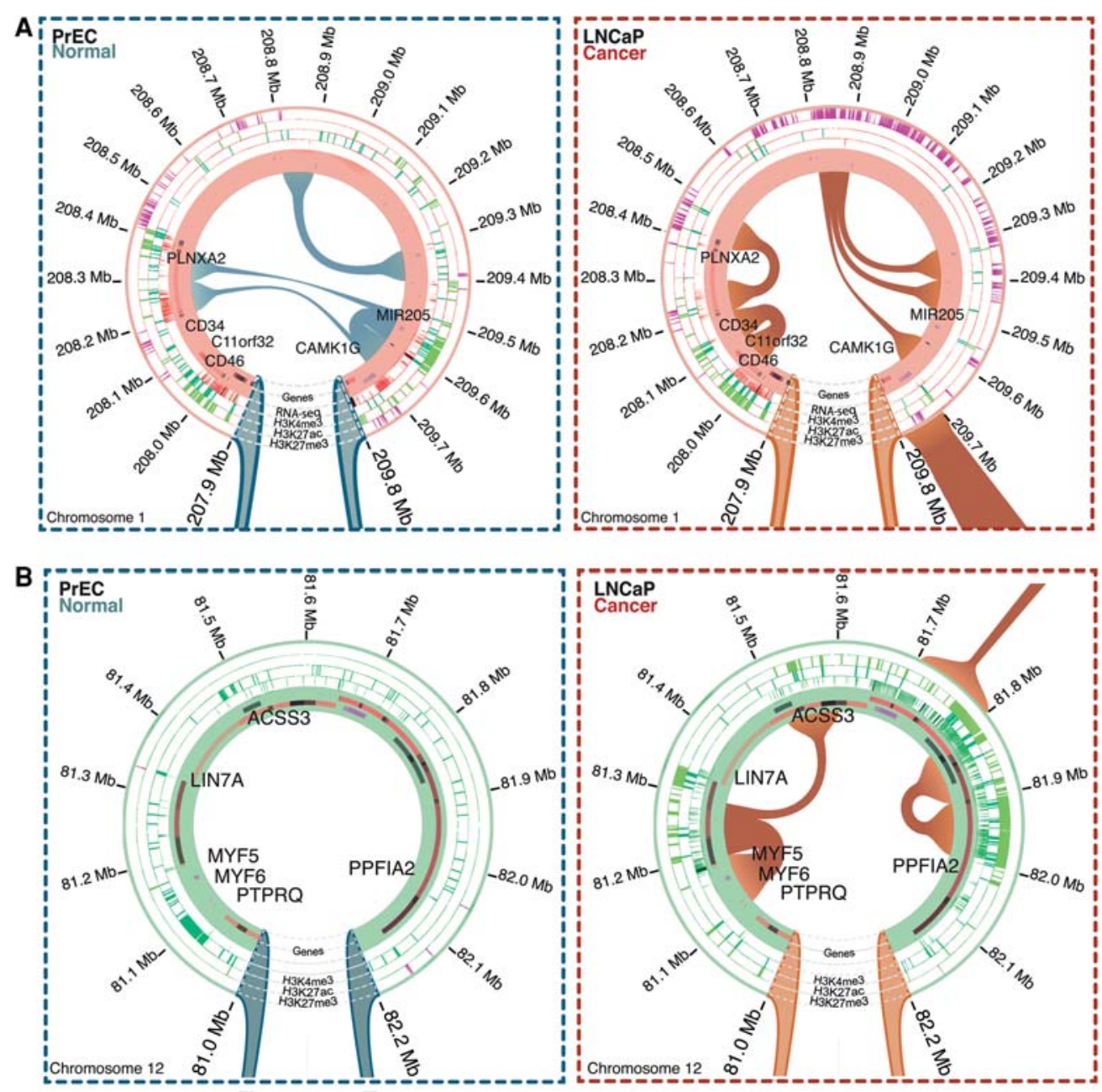

Figure 5. Long-range epigenetically silenced (LRES) and active (LREA) domains occur at differential interactions in cancer cells. $(A)$ A representative example of LRES domain on Chromosome 1, showing three chromatin interactions enriched for highly expressed genes (RNA-seq) and active histone marks in normal cells (H3K4me3 and H3K27ac). In cancer cells, new cancer-specific interactions are present in that region and are associated with loss of gene expression (RNA-seq), decrease in active histone marks, and increase in repressive marks (H3K27me3). (B) A representative example of LREA domain on Chromosome 12, showing the absence of chromatin interactions in normal cells that is associated with low levels of active histone marks (H3K4me3 and H3K27ac) and gene inactivity (RNA-seq). Aberrant, cancer-specific interactions in cancer cells result in high expression (RNA-seq) of genes and associated increase in active histone marks (H3K4me3 and H3K27ac). (Adapted, with permission, from Taberlay et al. 2016, (C) Cold Spring Harbor Laboratory Press.)

et al. 2007; Novak et al. 2008; Seng et al. 2008; Dallosso et al. 2009; Rafique et al. 2015). In prostate cancer, we have shown that LRES is primarily characterized by regional gains of repressive histone marks and loss of active histone marks encompassing tumor-suppressor or cancer-associated genes (Coolen et al. 2010). Regional activation of adjacent genes also occurs in cancer, and this is due to long-range epigenetic activation (LREA) (Bert et al. 2013). We found that LREA domains were associated with simultaneous gains in active histone marks and loss of repressive histone marks, and these domains commonly harbor oncogenes (Bert et al. 2013). Although these findings highlight the important role of longrange epigenetic remodeling in cancer, the potential impact on higher-order chromatin interactions has not been characterized.
To study the relationship between long-range epigenetic deregulation and $3 \mathrm{D}$ chromatin organization, we tested the significance of the intersection between LRES and LREA regions and cancer-specific differential interactions. We confirmed changes in gene expression between normal and cancer cell lines with RNA-seq. Using Ron$d o$, we visualized gene expression, histone modifications, and differential interactions overlapping known LRES and LREA domains. Figure 5A shows an example of differential interactions and epigenetic marks between normal PrECs and LNCaP cancer cells within a $\sim 1-\mathrm{Mb}$ region LRES region on chromosome 1. In normal cells, the LRES region that contains the genes PLXNA2, $M I R 205$, and $C A M K 1 G$ is actively expressed, as indicated by RNA-seq, whereas in cancer this region is silenced (Fig. 5A). Gene silencing across the LRES region is 
correlates with a gain of the repressive H3K27me3 mark and loss of the active $\mathrm{H} 3 \mathrm{~K} 4 \mathrm{me} 3$ and $\mathrm{H} 3 \mathrm{~K} 27 \mathrm{ac}$ chromatin marks (Fig. 5A). In Rondo we could observe a strong interaction between the active PLXNA2 and MIR205 loci in normal prostate cells. Notably, the chromatin interactions, spanning nearly $1 \mathrm{Mb}$, were located between putative active enhancers, marked by H3K27ac (Fig. 5A). A second interaction in the loci in the normal cells was observed in the intergenic region bounded by H3K27me3 marks. In contrast, in cancer cells, the chromatin interactions are notably altered. In fact the higher-order interactions between PLXNA2 with a putative enhancer element in normal cells are no longer observed. Instead, we find new local interactions and these are associated with loci that gain $\mathrm{H} 3 \mathrm{~K} 27 \mathrm{me} 3$. The data are consistent with the LRES region harboring inactive genes in a more condensed chromatin structure, and as such the epigenetically silenced MIR205 locus no longer interacts with the PLXNA2 locus in the cancer cells (Fig. 5A).

We also observed alterations in the $3 \mathrm{D}$ chromatin interactions in LREA domains and these too were associated with gene expression and epigenetic changes. Figure 5B shows an example of differential interactions and epigenetic marks between normal PrECs and LNCaP cancer cells within a $\sim 1.2-\mathrm{Mb}$ region LREA region on chromosome 12. These loci contain the genes PTPRQ, MYF6, MYF5, LIN7A, ACSS3, and PPFIA2, which in normal prostate cells are not expressed but in prostate cancer are concordantly activated (Fig. 5B). Regional gene activation across the LREA region is associated with gain of the active $\mathrm{H} 3 \mathrm{~K} 4 \mathrm{me} 3$ and $\mathrm{H} 3 \mathrm{~K} 27 \mathrm{ac}$ chromatin marks and new chromatin interactions between the MYF loci and LIN7A and LIN7A and ACSS3 (Fig. 5B). Notably, a new chromatin interaction at the PPFIA2 gene loci is also formed which intersects two potentially discrete enhancer regions enriched for H3K27ac (Fig. 5B). The data are consistent with the LREA region forming a more open chromatin structure and as such the epigenetically activated genes form new chromatin contacts with promoter and enhancer elements that are associated with concordant gene activation of the region. Together, these data indicate that long-range epigenetic dysregulation in cancer is also associated with an alteration in higher-order chromatin interactions commonly at regulatory loci.

\section{CONCLUSION}

Cancer is associated with widespread alterations to the genome and the epigenome. We propose that these changes are instrumental in the creation of a new 3D architecture that results in both localized and regional changes in gene expression. In contrast, the overall higher-order spatial organization of chromatin in cancer cells is highly similar to normal cells suggesting that on a large scale, chromosomes are folded similarly and retain their physical positions within the nucleus. Where the spatial organization of chromatin was altered in the cancer cells was restricted to the size of the TADs, which are smaller in cancer. Notably, these smaller TADs reside within the normal TAD architecture, rather than forming new TADs spanning domains. In fact, we found that the majority of domain boundaries that were present in normal cells were also present in cancer cells. We were surprised to find that more than half of the new smaller subdomain TADs that were acquired in both prostate cancer cell lines shared similar new boundaries. Clearly more chromatin conformation studies are required in many more cancer samples to determine whether there is indeed a cancer-specific process in 3D reorganization. Second, we identified new cancer-specific interactions within the smaller TADs. We found that these cancer differential interactions were enriched for regulatory elements (enhancers, promoters, and CTCF binding sites) suggesting that new chromatin interactions in cancer cells are involved in reprogramming the unique epigenetic programs of cancer cells. Enhancers are known to engage in interactions that exist in a cell typespecific manner and our results support the hypothesis that enhancer dynamics play a role in regulating local interaction differences between normal and cancer cells. Finally, we found that differential interactions also occur within long-range epigenetically deregulated domains in cancer cells. The regulatory regions within the long-range epigenetically deregulated domains show local changes in chromatin organization and abnormal chromatin interactions in both LRES and LREA regions. Of interest, we found new interactions at putative enhancers associated with gene activation in the cancer cells in LREA regions and loss of interactions at putative enhancer elements in cancer cells that are silenced in cancer in LRES regions.

In summary the implementation of a novel visualization tool has allowed us to explore $\mathrm{Hi}-\mathrm{C}$ interaction data in relation to the transcription and epigenetic changes between normal and prostate cancer cells. Our data support the hypothesis that alterations in the 3D chromatin state underpin the functional basis of the cancer genome by changing the spatial architecture of regulatory elements and proximity to genes resulting in aberrant cancer gene expression. Together our findings provide a new insight into long-range epigenetic dysregulation and higher-order chromatin interactions in cancer and suggests that long-range epigenetic dysregulation in cancer is associated with an alteration in higher-order chromatin interactions commonly at regulatory loci that potentially results in atypical promoter-enhancer and enhancer-enhancer interactions in cancer cells.

\section{ACKNOWLEDGMENTS}

S.J.C. is a National Health and Medical Research Council (NHMRC) Senior Principal Research Fellow \#1063559; P.C.T. is a NHMRC Career Development Fellow. This work was supported by grant \#1070613 and NHMRC Project grants \#1011447 and \#1051757.

\section{REFERENCES}

Achinger-Kawecka J, Clark SJ. 2017. Disruption of the 3D cancer genome blueprint. Epigenomics 9: 47-55. 
Andrey G, Montavon T, Mascrez B, Gonzalez F, Noordermeer D, Leleu M, Trono D, Spitz F, Duboule D. 2013. A switch between topological domains underlies HoxD genes collinearity in mouse limbs. Science 340: 1234167.

Barutcu AR, Lajoie BR, McCord RP, Tye CE, Hong D, Messier TL, Browne G, van Wijnen AJ, Lian JB, Stein JL, et al. 2015. Chromatin interaction analysis reveals changes in small chromosome and telomere clustering between epithelial and breast cancer cells. Genome Biol 16: 214.

Barutcu AR, Lian JB, Stein JL, Stein GS, Imbalzano AN. 2017. The connection between BRG1, CTCF and topoisomerases at TAD boundaries. Nucleus: 1-6.

Beagan JA, Gilgenast TG, Kim J, Plona Z, Norton HK, Hu G, Hsu SC, Shields EJ, Lyu X, Apostolou E, et al. 2016. Local genome topology can exhibit an incompletely rewired 3Dfolding state during somatic cell reprogramming. Cell Stem Cell 18: 611-624.

Bert SA, Robinson MD, Strbenac D, Statham AL, Song JZ, Hulf T, Sutherland RL, Coolen MW, Stirzaker C, Clark SJ. 2013. Regional activation of the cancer genome by long-range epigenetic remodeling. Cancer Cell 23: 9-22.

Boutros PC, Fraser M, Harding NJ, de Borja R, Trudel D, Lalonde E, Meng A, Hennings-Yeomans PH, McPherson A, Sabelnykova VY, et al. 2015. Spatial genomic heterogeneity within localized, multifocal prostate cancer. Nat Genet 47: 736-745.

Cancer Genome Atlas Research Network. 2015. The molecular taxonomy of primary prostate cancer. Cell 163: 1011-1025.

Coolen MW, Stirzaker C, Song JZ, Statham AL, Kassir Z, Moreno CS, Young AN, Varma V, Speed TP, Cowley M, et al. 2010. Consolidation of the cancer genome into domains of repressive chromatin by long-range epigenetic silencing (LRES) reduces transcriptional plasticity. Nat Cell Biol 12: $235-246$.

Dallosso AR, Hancock AL, Szemes M, Moorwood K, Chilukamarri L, Tsai HH, Sarkar A, Barasch J, Vuononvirta R, Jones $\mathrm{C}$, et al. 2009. Frequent long-range epigenetic silencing of protocadherin gene clusters on chromosome $5 \mathrm{q} 31$ in Wilms' tumor. PLoS Genet 5: e1000745.

Dekker J, Rippe K, Dekker M, Kleckner N. 2002. Capturing chromosome conformation. Science 295: 1306-1311.

Dixon JR, Selvaraj S, Yue F, Kim A, Li Y, Shen Y, Hu M, Liu JS, Ren B. 2012. Topological domains in mammalian genomes identified by analysis of chromatin interactions. Nature $\mathbf{4 8 5}$ : $376-380$.

Dixon JR, Jung I, Selvaraj S, Shen Y, Antosiewicz-Bourget JE, Lee AY, Ye Z, Kim A, Rajagopal N, Xie W, et al. 2015. Chromatin architecture reorganization during stem cell differentiation. Nature 518: 331-336.

Dostie J, Richmond TA, Arnaout RA, Selzer RR, Lee WL, Honan TA, Rubio ED, Krumm A, Lamb J, Nusbaum C, et al. 2006. Chromosome Conformation Capture Carbon Copy (5C): A massively parallel solution for mapping interactions between genomic elements. Genome Res 16: 12991309.

Dowen JM, Fan ZP, Hnisz D, Ren G, Abraham BJ, Zhang LN, Weintraub AS, Schuijers J, Lee TI, Zhao K, et al. 2014. Control of cell identity genes occurs in insulated neighborhoods in mammalian chromosomes. Cell 159: 374-387.

Ernst J, Kellis M. 2012. ChromHMM: Automating chromatinstate discovery and characterization. Nat Methods 9: 215-216.

Franke M, Ibrahim DM, Andrey G, Schwarzer W, Heinrich V, Schopflin R, Kraft K, Kempfer R, Jerkovic I, Chan WL, et al. 2016. Formation of new chromatin domains determines pathogenicity of genomic duplications. Nature 538: 265-269.

Frigola J, Song J, Stirzaker C, Hinshelwood RA, Peinado MA, Clark SJ. 2006. Epigenetic remodeling in colorectal cancer results in coordinate gene suppression across an entire chromosome band. Nat Genet 38: 540-549.

Fullwood MJ, Liu MH, Pan YF, Liu J, Xu H, Mohamed YB, Orlov YL, Velkov S, Ho A, Mei PH, et al. 2009. An oestrogen-receptor- $\alpha$-bound human chromatin interactome. Nature 462: $58-64$.
Giorgio E, Robyr D, Spielmann M, Ferrero E, Di Gregorio E, Imperiale D, Vaula G, Stamoulis G, Santoni F, Atzori C, et al. 2015. A large genomic deletion leads to enhancer adoption by the lamin B1 gene: A second path to autosomal dominant adult-onset demyelinating leukodystrophy (ADLD). Hum Mol Genet 24: 3143-3154.

Hitchins MP, Lin VA, Buckle A, Cheong K, Halani N, Ku S, Kwok CT, Packham D, Suter CM, Meagher A, et al. 2007. Epigenetic inactivation of a cluster of genes flanking MLH1 in microsatellite-unstable colorectal cancer. Cancer Res 67: 9107-9116.

Ibn-Salem J, Kohler S, Love MI, Chung HR, Huang N, Hurles ME, Haendel M, Washington NL, Smedley D, Mungall CJ, et al. 2014. Deletions of chromosomal regulatory boundaries are associated with congenital disease. Genome Biol 15: 423.

Kluth M, Harasimowicz S, Burkhardt L, Grupp K, Krohn A, Prien K, Gjoni J, Hass T, Galal R, Graefen M, et al. 2014. Clinical significance of different types of p53 gene alteration in surgically treated prostate cancer. Int J Cancer 135: $1369-$ 1380 .

Krijger PH, Di Stefano B, de Wit E, Limone F, van Oevelen C, de Laat W, Graf T. 2016. Cell-of-origin-specific 3D genome structure acquired during somatic cell reprogramming. Cell Stem Cell 18: 597-610.

Le Dily F, Bau D, Pohl A, Vicent GP, Serra F, Soronellas D, Castellano G, Wright RH, Ballare C, Filion G, et al. 2014. Distinct structural transitions of chromatin topological domains correlate with coordinated hormone-induced gene regulation. Genes Dev 28: 2151-2162.

Li Y, Huang W, Niu L, Umbach DM, Covo S, Li L. 2013. Characterization of constitutive CTCF/cohesin loci: A possible role in establishing topological domains in mammalian genomes. BMC Genomics 14: 553.

Lieberman-Aiden E, van Berkum NL, Williams L, Imakaev M, Ragoczy T, Telling A, Amit I, Lajoie BR, Sabo PJ, Dorschner $\mathrm{MO}$, et al. 2009. Comprehensive mapping of long-range interactions reveals folding principles of the human genome. Science 326: 289-293.

Lun AT, Smyth GK. 2015. diffHic: A Bioconductor package to detect differential genomic interactions in Hi-C data. $B M C$ Bioinformatics 16: 258.

Lupianez DG, Kraft K, Heinrich V, Krawitz P, Brancati F, Klopocki E, Horn D, Kayserili H, Opitz JM, Laxova R, et al. 2015. Disruptions of topological chromatin domains cause pathogenic rewiring of gene-enhancer interactions. Cell 161: $1012-1025$.

Misteli T. 2007. Beyond the sequence: Cellular organization of genome function. Cell 128: 787-800.

Nora EP, Lajoie BR, Schulz EG, Giorgetti L, Okamoto I, Servant $\mathrm{N}$, Piolot T, van Berkum NL, Meisig J, Sedat J, et al. 2012. Spatial partitioning of the regulatory landscape of the X-inactivation centre. Nature 485: 381-385.

Novak P, Jensen T, Oshiro MM, Watts GS, Kim CJ, Futscher BW. 2008. Agglomerative epigenetic aberrations are a common event in human breast cancer. Cancer Res 68: 8616-8625.

Phillips JE, Corces VG. 2009. CTCF: Master weaver of the genome. Cell 137: 1194-1211.

Phillips-Cremins JE, Sauria ME, Sanyal A, Gerasimova TI, Lajoie BR, Bell JS, Ong CT, Hookway TA, Guo C, Sun Y, et al. 2013. Architectural protein subclasses shape 3D organization of genomes during lineage commitment. Cell 153: $1281-1295$.

Pope BD, Ryba T, Dileep V, Yue F, Wu W, Denas O, Vera DL, Wang Y, Hansen RS, Canfield TK, et al. 2014. Topologically associating domains are stable units of replication-timing regulation. Nature 515: 402-405.

Rafique S, Thomas JS, Sproul D, Bickmore WA. 2015. Estrogen-induced chromatin decondensation and nuclear re-organization linked to regional epigenetic regulation in breast cancer. Genome Biol 16: 145.

Rao SS, Huntley MH, Durand NC, Stamenova EK, Bochkov ID, Robinson JT, Sanborn AL, Machol I, Omer AD, Lander ES, et al. 2014. A 3D map of the human genome at kilobase 
resolution reveals principles of chromatin looping. Cell 159: $1665-1680$

Seng TJ, Currey N, Cooper WA, Lee CS, Chan C, Horvath L, Sutherland RL, Kennedy C, McCaughan B, Kohonen-Corish MR. 2008. DLEC1 and MLH1 promoter methylation are associated with poor prognosis in non-small cell lung carcinoma. Br J Cancer 99: 375-382.

Simonis M, Klous P, Splinter E, Moshkin Y, Willemsen R, de Wit E, van Steensel B, de Laat W. 2006. Nuclear organization of active and inactive chromatin domains uncovered by chromosome conformation capture-on-chip (4C). Nat Genet 38: $1348-1354$.

Smith EM, Lajoie BR, Jain G, Dekker J. 2016. Invariant TAD Boundaries Constrain Cell-Type-Specific Looping Interactions between Promoters and Distal Elements around the CFTR Locus. Am J Hum Genet 98: 185-201.

Stransky N, Vallot C, Reyal F, Bernard-Pierrot I, de Medina SG, Segraves R, de Rycke Y, Elvin P, Cassidy A, Spraggon C, et al. 2006. Regional copy number-independent deregulation of transcription in cancer. Nat Genet 38: 1386-1396.

Symmons O, Uslu VV, Tsujimura T, Ruf S, Nassari S, Schwarzer W, Ettwiller L, Spitz F. 2014. Functional and topological characteristics of mammalian regulatory domains. Genome Res 24: 390-400.

Taberlay PC, Statham AL, Kelly TK, Clark SJ, Jones PA. 2014. Reconfiguration of nucleosome-depleted regions at distal regulatory elements accompanies DNA methylation of enhancers and insulators in cancer. Genome Res 24: 1421-1432.
Taberlay PC, Achinger-Kawecka J, Lun AT, Buske FA, Sabir K, Gould CM, Zotenko E, Bert SA, Giles KA, Bauer DC, et al. 2016. Three-dimensional disorganization of the cancer genome occurs coincident with long-range genetic and epigenetic alterations. Genome Res 26: 719-731.

Talagas M, Uguen A, Garlantezec R, Fournier G, Doucet L, Gobin E, Marcorelles P, Volant A, DE Braekeleer M. 2013. VEGFR1 and NRP1 endothelial expressions predict distant relapse after radical prostatectomy in clinically localized prostate cancer. Anticancer Res 33: 2065-2075.

Tsujimura T, Klein FA, Langenfeld K, Glaser J, Huber W, Spitz F. 2015. A discrete transition zone organizes the topological and regulatory autonomy of the adjacent tfap $2 \mathrm{c}$ and bmp7 genes. PLoS Genet 11: e1004897.

Williams JL, Greer PA, Squire JA. 2014. Recurrent copy number alterations in prostate cancer: An in silico meta-analysis of publicly available genomic data. Cancer Genet 207: 474488.

Zhang Y, McCord RP, Ho YJ, Lajoie BR, Hildebrand DG, Simon AC, Becker MS, Alt FW, Dekker J. 2012. Spatial organization of the mouse genome and its role in recurrent chromosomal translocations. Cell 148: 908-921.

Zhao Z, Tavoosidana G, Sjolinder M, Gondor A, Mariano P, Wang S, Kanduri C, Lezcano M, Sandhu KS, Singh U, et al. 2006. Circular chromosome conformation capture (4C) uncovers extensive networks of epigenetically regulated intra- and interchromosomal interactions. Nat Genet 38: $1341-1347$. 


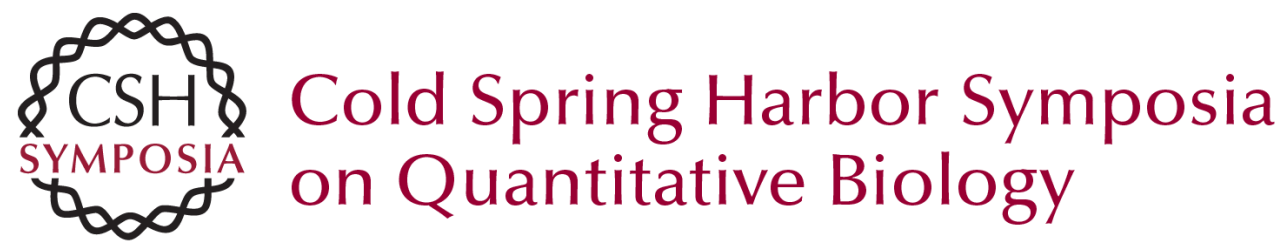

\section{Alterations in Three-Dimensional Organization of the Cancer Genome and Epigenome}

Joanna Achinger-Kawecka, Phillippa C. Taberlay and Susan J. Clark

Cold Spring Harb Symp Quant Biol 2016 81: 41-51 originally published online April 19, 2017 Access the most recent version at doi:10.1101/sqb.2016.81.031013

References This article cites 48 articles, 11 of which can be accessed free at: http://symposium.cshlp.org/content/81/41.full.html\#ref-list-1

Creative This article is distributed under the terms of the

Commons http://creativecommons.org/licenses/by/4.0/, which permits unrestricted

License reuse and redistribution provided that the original author and source are credited.

Email Alerting Receive free email alerts when new articles cite this article - sign up in Service the box at the top right corner of the article or click here. 